\title{
A GENERALIZED INDUCTIVE LIMIT TOPOLOGY FOR LINEAR SPACES
}

\author{
by S. O. IYAHEN and J. O. POPOOLA
}

(Received 24 September, 1971; revised 19 April, 1972)

1. In the usual definition of an inductive limit of locally convex spaces, one is given a linear space $E$, a family $\left(E_{\alpha}\right)$ of locally convex spaces and a set $\left(i_{\alpha}\right)$ of linear maps from $E_{\alpha}$ into $E$. Garling in [2] studies an extension of this, looking at absolutely convex subsets $S_{\alpha}$ of $E_{\alpha}$ and restrictions $j_{\alpha}$ of $i_{\alpha}$ to such sets. If, in the definition of Garling [2, p. 3], each $S_{\alpha}$ is instead a balanced semiconvex set, then the finest linear (not necessarily locally convex) topology on $E$ for which the maps $j_{\alpha}$ are continuous, will be referred to as the generalized $*$-inductive limit topology of the semiconvex sets. This topology is our object of study in the present paper; we find applications in the closed graph theorem.

2. The lemma on page 3 of [2] allows Garling to restrict attention to behaviour at the origin on the absolutely convex sets; Lemma 6.1 of [5] is a suitable replacement for our situation. An analogue of Theorem 2 of [2] can be proved and so also can the analogue of [5, Proposition 2.8, Corollary]. As in [2, p. 16, Example A], any separated almost convex ultrabornological space [5, Definitions 2.2 and 4.1$]$ has a generalized $*$-inductive limit topology (see [5, p. 303, paragraph 1]).

Let $E$ be a linear space of uncountable dimension. Let $\tau\left(E, E^{*}\right)$ and $s$, respectively, denote the finest locally convex topology and the finest linear topology on $E$. As the bounded subsets of $\left(E, \tau\left(E, E^{*}\right)\right)$ and $(E, s)$ are contained in finite-dimensional linear subspaces, $\tau\left(E, E^{*}\right)$ and $s$ induce the same topology on bounded sets. By $[2$, p. 16, Example A or B], $\left(E, \tau\left(E, E^{*}\right)\right)$ is the generalized inductive limit of a family of absolutely convex bounded sets. Clearly $(E, s)$ is the generalized *-inductive limit of this family. Since, by [9, Theorem 3.1], $\tau\left(E, E^{*}\right)$ is strictly coarser than $s$, we deduce that the complete separated bornological space $\left(E, \tau\left(E, E^{*}\right)\right)$ does not have a generalized *-inductive limit topology of bounded subsets. We also see from this example that the dual of a complete separated locally convex space under the topology of compact convergence need not be a generalized *-inductive limit space. However, in view of [2, Proposition 5 and p. 16, Example B], the dual of a Fréchet space under the topology of compact convergence has a generalized $*$-inductive limit topology, and so has any DF-space because of $[2$, p. 16, Example C].

The notion of a countably quasi-u.b. space, where u.b. is used as an abbreviation for ultra-barrelled, is introduced in [6, p. 609]. Such a space is called an ultra-DF space if it has a fundamental sequence of bounded sets. It follows from [6, Proposition 3.1] that the strong dual of any metrizable locally convex space is an ultra-DF space. The strict *-inductive limit of a sequence of separated ultra-DF spaces is ultra-DF. (To prove this, use the analogue for *-inductive limits of Theorem 2 (2) of [2], and [6, Theorem 3.1].)

Proposition 2.1. For each positive integer $i$, let $\mathscr{U}_{l}$ be a base of balanced neighbourhoods of the origin in a linear topological space $\left(E_{i}, \tau_{i}\right)$. Suppose that $S_{i}$ is a balanced semiconvex 
subset of $E_{i}$ and that $(E, \tau)$ is the generalized *-inductive limit of $\left(S_{i}\right)$ by one-to-one maps $t_{i}$. Then the family of sets

$$
\bigcup_{n \geqq 1} \sum_{i=1}^{n} t_{i}\left(U_{i} \cap S_{i}\right)
$$

as $U_{i}$ varies over $\mathscr{U}_{i}$, is a base of neighbourhoods of the origin in $(E, \tau)$.

Proof. Cf [5, Proposition 2.2].

Proposition 2.2. If an ultra-DF space $(E, \tau)$ has a fundamental sequence $\left(S_{n}\right)$ of balanced bounded sets such that, for some fixed $k \geqq 2$ and all $n, S_{n}+S_{n} \subseteq k S_{n}$, then $(E, \tau)$ necessarily has a generalized $*$-inductive limit topology.

Proof. Let $\left(S_{n}\right)$ be such a sequence; we may assume that it is increasing. Let $\tau^{\prime}$ be its generalized $*$-inductive limit topology.

For each $m=0,1,2, \ldots$, let $U^{m}$ be a balanced $\tau^{\prime}$-neighbourhood such that $U^{m+1}+$ $U^{m+1} \subseteq U^{m}$. By Proposition 2.1, we may suppose that $U^{m}$ is of the form

$$
\bigcup_{n \geqq 1} \sum_{i=1}^{n}\left(S_{i} \cap U_{i}^{m}\right)
$$

where each $U_{i}^{m}$ is a balanced $\tau$-neighbourhood, and the sequence $\left(U_{i}^{m}: m=0,1,2, \ldots\right)$ may be so chosen that

$$
U_{i}^{m+1}+U_{i}^{m+1} \subseteq U_{i}^{m}
$$

We are going to construct a bornivorous ultrabarrel $W^{0} \subseteq U^{0}$ of type $(\alpha)$ in $(E, \tau)$ (see [6, p. 609] for definition) and, since $(E, \tau)$ is countably quasi-u.b., it will follow that $U^{0}$ is a $\tau$-neighbourhood. We shall construct $W^{n} \subseteq U^{n}$ for each $n$.

With $n$ fixed, write $U$ for $U^{n}$ and $U^{\prime}$ for $U^{n+1}$, so that $U^{\prime}+U^{\prime} \subseteq U$; write $V_{i}$ for $U_{i}^{n+1}$ and $V_{i}^{\prime}$ for $U_{i}^{n+2}$, so that $V_{i}^{\prime}+V_{i}^{\prime} \subseteq V_{i}$. Thus $U$ and $U^{\prime}$ are $\tau^{\prime}$-neighbourhoods and $V_{i}, V_{i}^{\prime}$ are $\tau$-neighbourhoods, and $S_{i} \cap V_{i} \subseteq U^{\prime}$ for all $i$. Put

$$
r=k^{-n}, W_{i}={\overline{\left(r S_{i}\right) \cap U^{\prime}+V_{i}^{\prime}}}^{i} \text { and } W=\bigcap_{i \geqq 1} W_{i}
$$

If $x \in W$, then $x \in r S_{i}$ for some $i$, since $E=\bigcup_{i \geqq 1} r S_{i}$; hence, to show that $W \subseteq U$, it is enough to show that $\left(r S_{i}\right) \cap W_{i} \subseteq U$ for all $i$. Now $W_{i} \subseteq\left(r S_{i}\right) \cap U^{\prime}+V_{i}$ and so, if $x \in\left(r S_{i}\right) \cap W_{i}$, $x=y+z$, where $y \in\left(r S_{i}\right) \cap U^{\prime}$ and $z \in V_{i}$. Hence $x-y \in r k S_{i} \subseteq S_{i}$ and so $z \in S_{i} \cap V_{i} \subseteq U^{\prime}$. Hence $x \in U$. Now to show that $W$ is bornivorous, we show that $W$ absorbs each $S_{i}$. First $\left(r S_{i}\right) \cap U^{\prime}$ absorbs $S_{i}$ and, for $j \geqq i,\left(r S_{i}\right) \cap U^{\prime} \subseteq\left(r S_{j}\right) \cap U^{\prime} \subseteq W_{j}$, so that $\bigcap_{j \geqq l} W_{j}$ absorbs $S_{i}$. For $j<i, V_{j}^{\prime} \subseteq W_{j}$ and $V_{j}^{\prime}$ is a $\tau$-neighbourhood, and therefore $\bigcap_{j<i} W_{j}$ also absorbs $S_{i}$. 

then

Recall that, for each $n$, we put $U^{\prime}=U^{n+1}$ and $V_{i}^{\prime}$ for $U_{i}^{n+2}$. If we label such $W_{i}$ as $W_{i}^{n}$, and thus

$$
W_{i}^{n}+W_{i}^{n} \subseteq \overline{\left(k^{-(n-1)} S_{i}\right) \cap U^{n}+U_{i}^{n}}{ }^{\tau}=W_{i}^{n-1},
$$

$$
W^{n}+W^{n} \subseteq W^{n-1}
$$

Examples of $(E, \tau)$ covered by Proposition 2.2 include locally convex ultra-DF spaces and locally bounded spaces. If $H_{1}$ is the strong dual of a metrizable locally convex space and $\mathrm{H}_{2}$ is a separated locally bounded space that is not locally convex, then the product space $G=H_{1} \times H_{2}$ as well as the *-direct sum $[5$, p. 288] of countably many copies of $G$ satisfies the restrictions on $(E, \tau)$ (Use [5, Proposition 2.6]). We cannot as yet prove this result for almost convex ultra-DF spaces.

3. Let $(E, \xi)$ be a separated linear topological space (abbreviated to 1.t.s. in future) and $B$ a sequentially complete balanced semiconvex bounded subset of $(E, \xi)$. We shall denote by $\xi_{B}$ the complete separated locally bounded topology on the linear span $E_{B}$ of $B$ with the sequence $\left(n^{-1} B: n=1,2, \ldots\right)$ of sets as a base of neighbourhoods of the origin. Notice that $\xi_{B}$ is finer than the $\xi$-induced topology on $E_{B}$. Throughout this section, $(E, \tau)$ is the $*$-inductive limit of separated 1.t.s. $\left(E_{\alpha}, \tau_{\alpha}\right)$ by linear maps $t_{\alpha}$, where each $E_{\alpha}$ is spanned by a $\tau_{\alpha}$-sequentially. complete balanced semiconvex bounded set $S_{\alpha}$.

Let $T$ be a pointwise bounded set of continuous linear maps from $(E, \tau)$ into an 1.t.s. $H$. If $V$ is a closed balanced neighbourhood of the origin in $H$, then $T^{-1}(V)=\bigcap_{t \in T} t^{-1}(V)$ is an ultrabarrel in $(E, \tau)$. This implies that $t_{\alpha}^{-1}\left(T^{-1}(V)\right)$ is an ultrabarrel in $\left(E_{\alpha}, \tau_{\alpha}\right)$, and, by an application of Lemma 5.1 of [5], each set $T_{\circ} t_{\alpha}\left(S_{\alpha}\right)$ is bounded in $H$. (Cf $[8,12.4]$.)

PROPOSITION 3.1. Let a separated l.t.s. $(F, \xi)$ be the generalized *-inductive limit of a sequence $\left(C_{n}\right)$ of sequentially complete bounded subsets of $\left(F_{n}, \xi_{n}\right)$. If $T$ is a pointwise bounded set of linear maps from $(E, \tau)$ into $(F, \xi)$ such that the graph of each member of $T$ is closed in $(E, \tau) \times(F, \xi)$, then each set $T_{\circ} t_{\alpha}\left(S_{\alpha}\right)$ is bounded in $(F, \xi)$.

Proof. We may suppose that $F_{n}$ is the linear span of $C_{n}$. Write $\eta_{n}$ for $\xi_{c_{n}}$ and $\tau_{\alpha}^{\prime}$ for $\tau_{\alpha S_{\alpha}}$. If $(F, \eta)$ is the *-inductive limit of $\left(F_{n}, \eta_{n}\right)$, then $\eta$ is finer than $\xi$. It is sufficient to prove the proposition for the situation where $(E, \tau)$ is separated and spanned by some sequentially complete balanced semiconvex bounded set $S$. In this case, write $\tau_{0}$ for $\tau_{s}$. If $t$ is in $T$, the graph of $t$ is closed in $\left(E, \tau_{0}\right) \times(F, \eta)$. By Theorem 4.2 of [4] then, the map $t:\left(E, \tau_{0}\right) \rightarrow(F, \eta)$ is continuous. The result now follows from the remark preceding the statement of the proposition.

It is easily verified that the examples given in this paper of spaces with generalized *-inductive limit topologies satisfy the restriction imposed on $(E, \tau)$ if sequentially complete. Using the non-locally convex analogue of Theorem 2(2) of [2], we deduce from Proposition 3.1 that, if $(E, \tau)$ is the generalized *-inductive limit of an increasing sequence $\left(S_{n}\right)$ of complete bounded sets, where the topology of $S_{n}$ coincides with that induced by $S_{n+1}$, then the set $T$ is uniformly bounded on bounded sets. In particular, a closed linear map from $\operatorname{such}(E, \tau)$ into 
$(F, \xi)$ is bounded. However, if $G$ is the dual of a Fréchet space and $\lambda$ and $\mu$, respectively, are the topology on $G$ of compact convergence and the strong topology, the identity map from $(G, \lambda)$ into $(G, \mu)$ is closed but need not be continuous. Also, since $(G, \lambda)$ need not be quasibarrelled, the set $T$ in Proposition 3.1 need not be equicontinuous, even if each member of $T$ is continuous.

In addition to our original restriction on $(E, \tau)$, we shall in the following result assume that $(E, \tau)$ is the generalized *-inductive limit of $\left(S_{\alpha}\right)$.

Proposition 3.2. Let $(F, \xi)$ be a separated l.t.s. in which every closed bounded set is compact. If every linear map with a closed graph from any complete separated locally bounded space into $(F, \xi)$ is bounded, then a linear map from $(E, \tau)$ into $(F, \xi)$ is continuous if its graph is closed.

Proof. As in the proof of Proposition 3.1, we assume that $(E, \tau)$ is separated and spanned by some sequentially complete balanced semiconvex bounded set $S$, and write $\tau_{0}$ for $\tau_{s}$.

The graph of $f$ is necessarily closed in $\left(E, \tau_{0}\right) \times(F, \xi)$, and therefore the closure $C$ of $f(S)$ in $(F, \xi)$ is compact, being bounded. Also, because the graph of $f$ is closed in $(E, \tau) \times(F, \xi)$, for some separated linear topology $\lambda$, say, on $F$ coarser than $\xi$, the map $f:(E, \tau) \rightarrow(F, \lambda)$ is continuous. The identity map $(C, \xi) \rightarrow(C, \lambda)$, being continuous, is a homeomorphism. Therefore the restriction of $f$ to $(S, \tau) \rightarrow(F, \xi)$ is continuous. This implies that $f:(E, \tau) \rightarrow(F, \xi)$ is continuous, since $(E, \tau)$ is the generalized *-inductive limit of $\left(S_{\alpha}\right)$.

- The above result is not true if $(E, \tau)$ is as in Proposition 3.1. For, let $H$ be the dual of a reflexive Banach space of infinite dimension and $\lambda$ and $\mu$, respectively, the weak topology on $H$ and the topology of compact convergence. The identity map from $(H, \lambda)$ into $(H, \mu)$ is closed but not continuous. The space $(H, \mu)$ satisfies the conditions on $(F, \xi)$ in Proposition 3.2; $(H, \lambda)$ is spanned by an absolutely convex compact set and is the inductive limit of $(H, \lambda)$ by the identity map $(H, \lambda) \rightarrow H$.

In Proposition 3.2, $(F, \xi)$ could be the generalized *-inductive limit of an increasing sequence of compact sets, by Proposition 3.1, or a Montel-Fréchet or LF space, or a sequence space $l\left(p_{n}\right)$ of $[1$, Corollary 2.3] or the strict $*$-inductive limit $[5$, p. 290] of a sequence of such spaces (See Theorem 4.2 of [4]). The space $(E, \tau)$ could be a separated sequentially complete almost convex ultrabornological space or the dual of a Fréchet space under the topology of compact convergence or the type in Proposition 2.2, provided that it is sequentially complete. If, in addition, $(E, \tau)$ is locally convex (in which case it has a generalized inductive limit topology in the sense of [2]), then, by [10], $(F, \xi)$ could be any of the distribution spaces $\mathscr{E}, \mathscr{E}^{\prime}, \mathscr{D}, \mathscr{D}^{\prime}, \mathscr{S}, \mathscr{S}^{\prime}, \mathcal{O}_{M}, \mathcal{O}_{M}^{\prime}, \mathcal{O}_{C}, \mathcal{O}_{C}^{\prime}$. A different technique is employed in [3] to obtain a similar result where $(E, \tau)$ is the generalized inductive limit of compact sets.

Proposition 3.3. If $(F, \xi)$ is any of the above ten distribution spaces or a reflexive Fréchet or LF space and $(E, \tau)$ is the strong dual of a metrizable locally convex space, then a closed linear map $t$ from $(E, \tau)$ into $(F, \xi)$ is necessarily continuous.

Proof. If $\xi_{0}$ is the weak topology associated with $\xi$, the graph of $t$ must be closed in $(E, \tau) \times\left(F, \xi_{0}\right)$, by $[8,17.1]$. Therefore the map $t:(E, \tau) \rightarrow\left(F, \xi_{0}\right)$ is continuous, and, by $[8,21.43]$, the result then follows.

Notice that, in the above, $(E, \tau)$ need not be barrelled. 
The closed graph theorem does not hold for linear maps from $E$ into $F$ if $E$ is a u.b. space (See [6, p. 609]) and $F$ is the generalized $*$-inductive limit of a sequence of complete absolutely convex bounded sets. To see this, let $(H, \eta)$ be an incomplete separated inductive limit of an increasing sequence of Banach linear subspaces. By taking for our defining sets scalar multiples of the unit balls in the Banach spaces, one can express $(H, \eta)$ as the generalized *-inductive limit of some sequence of complete absolutely convex bounded sets. As $(H, \eta)$ is u.b. [5, Theorem 3.2, Corollary 2] but not $B_{r}$-complete, there is a separated u.b. topology $\mu$, say, on $H$ coarser than $\eta$ [4, Proposition 2.3]. The identity map from $(H, \mu)$ into $(H, \eta)$ is not continuous, though it has a closed graph. We do not know if the closed graph theorem holds for linear maps from u.b. spaces to generalized *-inductive limits of sequences of compact sets. We now introduce a class of spaces for which this result is true. The idea is to generalize the notion of an ultrabarrel in a way similar to that in which the concept of a barrel is extended in [7].

4. Let $B$ be a suprabarrel in an l.t.s. $E$ and $\left(B_{n}\right)$ a defining sequence for $B$ [5, Definition 3.1]. Suppose that, for each $m, B_{m}$ contains some $\bigcup_{n \geqq 1} A_{n}^{m}$, where $A_{n}^{m}$ is a closed balanced subset of $E$ and $\bigcup_{n \geqq 1} A_{n}^{m}$ is absorbent. We shall call such a $B$ an $F_{\sigma}$-ultrabarrel. An 1.t.s. $E$ is known as an $F_{\sigma}$-u.b. space if, in $E$, every $F_{\sigma}$-ultrabarrel is a neighbourhood of the origin.

Proposition 4.1. Let a separated l.t.s. $(F, \xi)$ be the generalized *-inductive limit of an increasing sequence $\left(C_{n}\right)$ of compact subsets. If $E$ is an $F_{\sigma}$-u.b. space, then a linear map $t$ from $E$ into $(F, \xi)$ is continuous if its graph is closed.

Proof. Let $V$ be a balanced $\xi$-neighbourhood in $F$. As each $C_{n}$ is $\xi$-bounded, $r_{n} C_{n} \subseteq V$ for some positive real number $r_{n}$. The set $\bigcup_{n \geqq 1} r_{n} C_{n}$ is absorbent in $F$, since $\bigcup_{n \geqq 1} C_{n}=F$. If the graph of $t$ is closed in $E \times(F, \xi)$, there is a separated linear topology $\lambda$, say, on $F$ coarser than $\xi$ such that the map $t: E \rightarrow(F, \lambda)$ is continuous. Each $r_{n} C_{n}$ being $\xi$-compact is $\lambda$-compact and thus closed in $(F, \lambda)$. One shows in this way that $V$ is an $F_{\sigma}$-ultrabarrel in $(F, \lambda)$, and, from the continuity of the map $t: E \rightarrow(F, \lambda), t^{-1}(V)$ is then an $F_{\sigma}$-ultrabarrel in the $F_{\sigma}$-u.b. space $E$. Thus $t^{-1}(V)$ is a neighbourhood in $E$, and this gives the result.

An 1.t.s. $E$ of the second category is necessarily an $F_{\sigma}$-u.b. space. For, if $B$ is an $F_{\sigma}$-ultrabarrel in $E$ with $\left(B_{n}\right)$ as a defining sequence, then $B_{1}$ contains some $\bigcup_{n \geqq 1} A_{n}^{1}$. As this is absorbent, $E=\bigcup_{m, n \geq 1} m A_{n}^{1}$, and, since $E$ is of the second category, there are positive integers $M, N$ such that the interior (with respect to $E$ ) of $M A_{N}^{1}$ is not empty. From this, one shows that $A_{N}^{1}+A_{N}^{1} \subseteq B$ is a neighbourhood of the origin. One can prove that a $*$-inductive limit of $F_{\sigma}$-u.b. spaces is an $F_{\sigma}$-u.b. space. In particular, an LF space or the *-direct sum of any family of complete metric linear spaces is an $F_{\sigma}$-u.b. space.

Clearly, any $F_{\sigma}$-u.b. space is u.b. But not every u.b. space is an $F_{\sigma}$-u.b. space. To show this, in view of [5, p. 299, paragraph 2], it is sufficient to prove that an $F_{a}$-u.b. space $H$ with a fundamental sequence $\left(D_{n}\right)$ of closed balanced bounded sets is ultrabornological. If $B$ is a suprabarrel in $H$ with a defining sequence $\left(B_{n}\right)$ of bornivorous sets, then, for each $n$, there is a 
sequence $\left(S_{m}^{n}: m=1,2, \ldots\right)$ of positive real numbers such that $\bigcup_{m \geq 1} S_{m}^{n} D_{m} \subseteq B_{n}$. Thus $B$ is a neighbourhood in $H$, being an $F_{\sigma}$-ultrabarrel. By [5, Lemma 4.1] then, $H$ is ultrabornological.

Proposition 4.1 is however true for some $E$ not an $F_{\sigma}$-u.b. space. Since $(F, \xi)$ is complete, if $G_{0}$ is a dense linear topological subspace of $G$, then the closed graph theorem holds for linear maps from $G$ into $(F, \xi)$ if it holds for linear maps from $G_{0}$ into $(F, \xi)$. Thus, if $E_{1}$ is as in [5, p. 299, paragraph 2], any closed linear map from $E_{1}$ into $(F, \xi)$ is continuous.

The authors wish to thank the referee for helpful suggestions.

\section{REFERENCES}

1. B. A. Barnes and A. K. Roy, Boundedness in certain topological linear spaces, Studia Math. 33 (1969), 147-156.

2. D. J. H. Garling, A generalized form of inductive limit topology for vector spaces, Proc. London Math. Soc. (3)14 (1964), 1-28.

3. S. O. Iyahen, A closed graph theorem; to appear.

4. S. O. Iyahen, $D(\tau ; l)$-spaces and the closed graph theorem, Proc. Edinburgh Math. Soc. 16 (1969), 89-99.

5. S. O. Iyahen, On certain classes of linear topological spaces, Proc. London Math. Soc. (3) 18 (1968), 285-307.

6. S. O. Iyahen, On certain classes of linear topological spaces II, J. London Math. Soc. (2) 3 (1971), 609-617.

7. S. O. Iyahen, The domain space in a closed graph theorem II, Rev. Roum. Math. Pures et Appl. 17 (1972), 39-46.

8. J. L. Kelley and I. Namioka, Linear topological spaces (New York, 1963).

9. V. L. Klee, Convex sets in linear spaces III, Duke Math. J. 20 (1953), 105-111.

10. L. Schwartz, Sur le théorème du graphe fermé, C.R. Acad. Sci. Paris Séries A-B 263 (1966), $A 602-A 605$.

UNIVERSITY OF BENIN,

College of Education, BENIN CITY, NIGERIA UNIVERSITY OF LAGOS, LAGOS, Nigeria 